\title{
High energy particle collisions near black holes
}

\author{
O. B. Zaslavskii ^ \\ ${ }^{1}$ Department of Physics and Technology, Kharkov V.N. Karazin National University, 4 Svoboda Square, \\ Kharkov 61022, Ukraine
}

\begin{abstract}
If two geodesic particles collide near a rotating black hole, their energy in the centre of mass frame $E_{c . m}$. can become unbound under certain conditions (the so-called BSW effect). The special role is played here by so-called critical geodesics when one of particles has fine-tuned energy and angular momentum. The nature of geodesics reveals itself also in fate of the debris after collisions. One of particles moving to a remote observer is necessarily near-critical. We discuss, when such a collision can give rise not only unboud $E_{c . m}$. but also unbound Killing energy E (so-called super-Penrose process).
\end{abstract}

Investigation of high energy collisions of particles near black holes comes back to works [1] [3]. In recent years, an interest to this issue revived after the observation made by Bañados, Silk and West (the BSW effect, after the names of its authors) that particle collision near the Kerr black hole can lead, under certain additional conditions, to the unbounded growth of the energy in their centre of mass $E_{c . m .}$. [4]. Later on, in a large series of works, this observation was generalized and extended to other objects and scenarios. The energy that appears in the BSW effect is relevant for an observer who is present just near the point of collision in the vicinity of the black hole horizon. Meanwhile, what is especially physically important is the Killing energy $E$ of debris after such a collision measured by an observer at infinity. Strong redshift "eats" significant part of $E_{c . m}$., so it was not quite clear in advance, to what extent the energy $E$ may be high. If $E$ exceeds the initial energy of particles, we are faced with the energy extraction from a black hole. This is a so-called collisional Penrose process (the Penrose process that occurs due to particle collisions). Thus there are two related but different issues: (i) investigation of the effect of unbounded $E_{c . m}$., (ii) study of properties of $E$ and the question about the maximum possible efficiency of the collisional Penrose process.

Consider the generic axially symmetric metric. It can be written as

$$
d s^{2}=-N^{2} d t^{2}+g_{\phi \phi}(d \phi-\omega d t)^{2}+d l^{2}+g_{z z} d z^{2} .
$$

Here, the metric coefficients do not depend on $t$ and $\phi$. On the horizon $N=0$. Alternatively, one can use coordinates $\theta$ and $r$, similar to Boyer-Lindquist ones for the Kerr metric, instead of $l$ and $z$. In (1) we assume that the metric coefficients are even functions of $z$, so the equatorial plane $\theta=\frac{\pi}{2}(z=0)$ is a symmetry one.

In the space-time under discussion there are two conserved quantities $u_{0} \equiv-E$ and $u_{\phi} \equiv L$ where $u^{\mu}=\frac{d x^{\mu}}{d \tau}$ is the four-velocity of a test particle, $\tau$ is the proper time and $x^{\mu}=(t, \phi, l, z)$ are coordinates..The aforementioned conserved quantities have the physical meaning of the energy per unit mass (or frequency for a lightlike particle) and azimuthal component of the angular momentum,

\footnotetext{
^e-mail: zaslav@ukr.net
} 
respectively. It follows from the symmetry reasonings that there exist geodesics in such a background which lie entirely in the plane $\theta=\frac{\pi}{2}$. We consider a pair of particles labeled by the subscript $i=1,2$ and having the equal rest masses $m_{1}=m_{2}=m$. We also assume that both particles are approaching the horizon. Then, using the first integrals for such geodesics one can calculate the energy in the centre of mass frame $E_{c . m .}=\sqrt{2} m \sqrt{1-u_{\mu(1)} u^{\mu(2)}}$ [4]. After simple manipulations, one obtains

$$
\frac{E_{c . m .}^{2}}{2 m^{2}}=\frac{X_{1} X_{2}-Z_{1} Z_{2}}{N^{2}}+1-Y=\frac{L_{1} L_{2}}{g_{\phi \phi}},
$$

where $X=X_{i} \equiv E_{i}-\omega L_{i}$,

$$
Z_{i}=\sqrt{\left(E_{i}-\omega L_{i}\right)^{2}-N^{2} b_{i}}, b_{i}=1+\frac{L_{i}^{2}}{g_{\phi \phi}} .
$$

In the horizon limit $N \rightarrow 0$ one obtains

$$
\left(\frac{E_{c . m .}^{2}}{2 m^{2}}\right)_{H}=1+\frac{b_{1(H)}\left(L_{2(H)}-L_{2}\right)}{2\left(L_{1 H}-L_{1}\right)}+\frac{b_{2(H)}\left(L_{(1) H}-L_{1}\right)}{2\left(L_{2(H)}-L_{2}\right)}-\frac{L_{1} L_{2}}{\left(g_{\phi \phi}\right)_{H}}, L_{i(H)} \equiv \frac{E_{i}}{\omega_{H}} .
$$

By a very meaning of derivation, it is supposed in (4) that $L_{1} \neq L_{2(H)}, L_{2} \neq L_{2(H)}$. If $L_{1}=L_{1(H)}(1-\varepsilon)$, $\varepsilon \ll 1, L_{2} \neq L_{2(H)}$,

$$
\left(\frac{E_{c . m .}^{2}}{2 m^{2}}\right)_{H} \approx \frac{b_{1(H)}\left(L_{2(H)}-L_{2}\right)}{\left.2 L_{1(H}\right) \varepsilon}
$$

becomes unbounded. This is just the BSW effect. What is important is that the effect is universal and takes place near generic rotating axially symmetric black holes [6]. Moreover, this happens near not only extremal black holes (as assumed usually) but near nonextremal one as well [7].

One can suggest a quite generic explanations based on geometric properties of the particles' trajectories near the horizon [8]. Let us consider the collision of two particles near the future horizon of a black hole. We assume that both particles move towards the horizon. Let us introduce in the point $P$ under consideration and its vicinity the tetrad with lightlike vectors $l^{\mu}, N^{\mu}$ and spacelike vectors $a^{\mu}$, $b^{\mu}$ orthogonal to them. Here, the vectors $l^{\mu}, N^{\mu}$ are normalized, say, as $l^{\mu} N_{\mu}=-1$.

Then,

$$
g_{\alpha \beta}=-l_{\alpha} N_{\beta}-l_{\beta} N_{\alpha}+\sigma_{\alpha \beta}
$$

where $\sigma_{\alpha \beta}=a_{\alpha} b_{\beta}+a_{\beta} b_{\alpha}, l^{\alpha} \sigma_{\alpha \beta}=N^{\alpha} \sigma_{\alpha \beta}=0$. We assume that it is the vector $l^{\mu}$ that becomes the generator of the future horizon. In general, we can use the decomposition of the four-velocity $u^{\mu}$ in the form

$$
u_{i}^{\mu}=\frac{l^{\mu}}{2 \alpha_{i}}+\beta_{i} N^{\mu}+s_{i}^{\mu}, s_{i}^{\mu}=A_{i} a^{\mu}+B_{i} b^{\mu}
$$

where $i=1,2$ labels the particles and $\alpha_{i}, \beta_{i}, A_{i}$ and $B_{i}$ are coefficients. The time-like vector $u^{\mu}$ is normalized as usual, $(u u)=-1$, hereafter the symbol (...) denotes the scalar product. Then, it follows from (7) that

$$
\begin{gathered}
\beta_{i}=-\left(u_{i} l\right), \\
\alpha_{i}=-\frac{1}{2}\left(u_{i} N\right)^{-1} .
\end{gathered}
$$

As vectors $u^{\mu}, l^{\mu}, N^{\mu}$ are assumed to be future-directed, $\alpha_{i}>0, \beta_{i}>0$ (motion "forward in time"). The normalization condition entails

$$
s_{i}^{\mu} s_{i \mu}=\frac{\beta_{i}}{\alpha_{i}}-1
$$


The case $\beta_{i}=\alpha_{i}, s_{i}^{\mu}=0$ corresponds to pure radial motion (see below).

Then,

$$
-\left(u_{1} u_{2}\right)=\frac{1}{2}\left(\frac{\beta_{1}}{\alpha_{2}}+\frac{\beta_{2}}{\alpha_{1}}\right)-\left(s_{1} s_{2}\right) .
$$

The energy in the centre of mass frame [4] is equal to $E_{c . m .}^{2}=m_{1}^{2}+m_{2}^{2}-2 m_{1} m_{2}\left(u_{1} u_{2}\right)\left(m_{i}\right.$ are rest masses of particles), so

$$
E_{c . m .}^{2}=m_{1}^{2}+m_{2}^{2}+m_{1} m_{2}\left[\frac{\beta_{1}}{\alpha_{2}}+\frac{\beta_{2}}{\alpha_{1}}-2\left(s_{1} s_{2}\right)\right] .
$$

The frame of the centre of mass falls down with both particles [4], so the possible effect of unbound acceleration is not direct manifestation of the blueshift. In general, as it is seen from (12), $E_{c . m}^{2}$. remains finite even in the vicinity of the horizon for any nonzero $\alpha_{1}, \alpha_{2}$. However, if, say, $\alpha_{1} \rightarrow 0$ that corresponds to a so-called critical particle, $E_{c . m .}^{2} \rightarrow \infty$.

We can also suggest simple kinematic explanation. We consider the geodesic motion of massive particles in the equatorial plane $\theta=\frac{\pi}{2}$. Then, the relation holds [9]

$$
E-\omega L=\frac{N}{\sqrt{1-V^{2}}}
$$

where $V$ is the velocity measured by a local zero angular momentum observer (ZAMO) [5]. One can observe that for generic $E, L$ in the horizon limit $N \rightarrow 0$ the velocity $V \rightarrow 1$. But for $E=\omega_{H} L\left(\omega_{H}\right.$ is the horizon value), $V<1$. Thus we have collision between one "slow" and one "rapid" particles. Their relative velocity $w \rightarrow 1$, the Lorentz factor of relative motion $\gamma=\frac{1}{\sqrt{1-w^{2}}} \rightarrow \infty$, the energy $E_{\text {c.m. }} \rightarrow \infty$.

Now, we turn to the question, what can see an observer at infinity. If $E$ exceeds the initial energy of particles, we are faced with the energy extraction from a black hole. This is a so-called collisional Penrose process (the Penrose process that occurs due to particle collisions). Strong redshift "eats" significant part of $E_{c . m}$, so it was not quite clear in advance, to what extent the energy $E$ may be high. It turned out that energy extraction from the Kerr black hole is possible but it was find to be relatively modest [10], [11], [12]. More general situation not restricted to the Kerr metric was considered in [13] where quite general upper bounds were derived that depend on the details of the metric. But, also, indefinitely large $E$ turned out to be impossible.

The aforementioned results on the energy extraction were obtained for the standard BSW scenario: both colliding particles move towards a black hole, one of which is "critical" with fine-tuned parameters (energy and angular momentum), the second particle is "usual" (not fine-tuned). However, numeric findings in [16] showed that if the critical particle moves away from a black hole, the efficiency of the process significantly increases and amounts to 13,92 for the Kerr metric. In what follows, we call this Schnittman process (scenario). Later, numerically [17] and analytically [18] it was found that if colliding particles move in opposite directions and both of them are usual, a formally infinite efficiency becomes possible (so-called super-Penrose process). The problem is, however, that a usual particle with a finite energy cannot move away from a black hole although for the critical particle this is possible ([10], [13], [14]). Therefore, one could think that an outgoing usual particle could be created in some precedent collision. However, more careful treatment showed that the kind of particles under discussion cannot appear as a result of preceding collisions with finite masses and angular momenta. For a divergent mass of an initial particle this is possible but this reduces the physical value of the process [19], [20]. One is led to conclusion that starting from initial conditions in which usual outgoing particles near the black hole horizon are absent one cannot obtain them by means of additional collisions. As a result, a super-Penrose process near black holes is impossible. 
Account for more involved scenarios in which particles intermediate between the critical and usual ones participate, only confirmed this conclusion [14]. (There is another option when collision occurs near a white black hole [15] but we do not discuss here these rather exotic objects.)

Quite recently, the estimates of the efficiency of the energy extraction from the Kerr black hole found in [16] numerically were derived analytically for the Kerr metric [21], [22]. General approach was developed in [23]. Maximum efficiency of extraction in the Kerr metric requires heavy particles that fall into a black hole [22] but for more general metrics this is not necessarily so [23].

What is especially important is that extraction can become infinite if instead of a black hole collision happens in the background without the horizon but on the threshold of its formation. This was shown in a general form in [24] and analyzed in detail in [25].

It turns out that collisions between ingoing and outgoing particles near generic axially symmetric blakc holes lead to some new qualitative possibilities absent for the Kerr one. In particular, the maximum efficiency of the energy extraction becomes possible even without heavy produced particles that are necessary in the Kerr case [22].

The conservation of the energy and angular momentum gives us

$$
E_{1}+E_{2}=E_{3}+E_{4}, L_{1}+L_{2}=L_{3}+L_{4}
$$

Let a particle move towards a centre, bounce back and collide with another ingoing particle. Examination of (14) in the background (1), where $N>0$ but is small at some $r_{0}$ shows that there are scenarios in which formally $E_{3}, L_{3} \rightarrow \infty$ and $E_{4}, L_{4} \rightarrow-\infty$. Thus extraction becomes unbounded. One reservation is in order. All particles are considered in the test approximation, so self-gravitation is neglected. There exists another mechanism of the energy extraction related to the electrically charged particles [26], [27] but we did not discuss it here.

How to take into account the effect of self-gravitaiton for particle collision under discussion remains unresolved very interesting problem.

Acknowledgments. I thank organizers for support that made my participation in the seminar possible.

\section{References}

[1] T. Piran, J. Katz, and J. Shaham, Astrophys. J. 196, L107 (1975).

[2] T. Piran and J. Shaham, Astrophys. J. 214, 268 (1977).

[3] T. Piran and J. Shanam, Phys. Rev. D 16, 1615 (1977).

[4] M. Bañados, J. Silk and S.M. West, Phys. Rev. Lett. 103 (2009) 111102.

[5] J. M. Bardeen, W. H. Press, and S. A. Teukolsky, Astrophys. J. 178, 347 (1972).

[6] O.B. Zaslavskii, Phys. Rev. D 82 (2010) 083004.

[7] A.A. Grib and Yu.V. Pavlov, Pis'ma v ZhETF 92, 147 (2010) [JETP Letters 92, 125 (2010)].

[8] O. B. Zaslavskii, Class. Quantum Grav. 28, 105010(2011).

[9] O. B. Zaslavskii, Phys. Rev. D 84, 024007 (2011)

[10] T. Harada, H. Nemoto and U. Miyamoto, Phys. Rev. D 86 (2012) 024027.

[11] M. Bejger, T. Piran, M. Abramowicz, and F. Håkanson, Phys. Rev. Lett. 109 (2012) 121101.

[12] T. Harada, K. Ogasawara, U. Miyamoto, [arXiv:1606.08107].

[13] O. Zaslavskii, Phys. Rev. D 86 (2012) 084030.

[14] O. B. Zaslavskii, Phys. Rev. D 93, 024056 (2016).

[15] A. A. Grib and Yu. V. Pavlov, Gravitation and Cosmology 21, 13 (2015).

[16] J. D. Schnittman, Phys.Rev.Lett. 113, 261102 (2014).

[17] E. Berti, R. Brito and V. Cardoso, Phys. Rev. Lett. 114, 251103 (2015). 
[18] O. Zaslavskii, Mod. Phys. Lett. A 30 (2015) 1550076.

[19] E. Leiderschneider and T. Piran, arXiv:1501.01984.

[20] O. B. Zaslavskii, Europhys. Lett. 11150004 (2015).

[21] E. Leiderschneider, T. Piran, Phys. Rev. D 93, 043015 (2016), [arXiv:1510.06764].

[22] K. Ogasawara, T. Harada, U. Miyamoto, Phys. Rev. D 93, 044054 (2016).

[23] O. B. Zaslavskii, [arXiv:1607.00651].

[24] O. B. Zaslavskii, Phys. Rev. D 88, 044030 (2013).

[25] M. Patil, T. Harada, Ken-ichi Nakao, P. S. Joshi, M. Kimura, Phys. Rev. D 93, 104015 (2016).

[26] O. B. Zaslavskii, Phys. Rev. D 86, 124039 (2012).

[27] H. Nemoto, U. Miyamoto, T. Harada, and T. Kokubu, Phys. Rev. D 87, 127502 (2013). 